AGRICULTURE AND BIOLOGY JOURNAL OF NORTH AMERICA

ISSN Print: 2151-7517, ISSN Online: 2151-7525, doi:10.5251/abjna.2013.4.3.309.315

(C) 2013, ScienceHuß, http://www.scihub.org/ABJNA

\title{
Reaction of certain malvaceae cultivars to meloidogyne incognita infection under greenhouse conditions
}

\author{
Gad, S. B.; A. G. El-Sherif and A. R. Refaei \\ Nematology Res. Unit, Agric. Zool. Dept, Fac. of Agric., Mansoura Univ. Dk. Egypt. \\ Corresponding author : EL-Sherif, A. G. E-mail : elsherifmohammed@yahoo.com
}

\begin{abstract}
A greenhouse experiment was conducted to study the reaction of nine malvaceae cultivars i.e. cotton cvs. Giza 86, 70, 90 and 89; okra, cvs Hyper Doki 1\& 2 and Ismaily; and rosella, cvs. Hardy and Sabahia 17 to Meloidogyne incognita infection through certain plant growth parameters and nematode criteria. Results indicated that all tested plant growth parameters were obviously reduced by $M$. incognita infection at various degrees. Of the tested Malvaceae, plant growth parameters such as total plant fresh weights showed the highest reduction percentage values appointed 36.19 and $34.32 \%$ by cotton cvs. Giza 90\& Giza 86 ; 32.41\% for okra cv. Hyper Dokki 1 and $31.54 \%$ by rosella cv, Sabahia17, respectively. Meanwhile, their least values of the same criterion assigned 18.70 and $19.81 \%$ for cotton cvs, Giza 89 \& Giza 70 and $21.81 \%$ for okra cv. Ismaily, respectively. Due to the relationship between reduction percentage of plant growth and reproduction factor (RF), Okra cv. Ismaily and rosella cv. Hardy were classified as moderately resistant and intolerant hosts, respectively, whereas cotton cvs. Giza 90 \& 86 ; Okra cv. Hyper Dokki 1 and rosella cv. Sabahia 17 were rated as highly susceptible hosts. Meanwhile, cotton cv. Giza 89 \& 70 and Okra cv. Hyper Dokki 2 were scored as susceptible hosts to $M$. incognita infection, since their percentage reduction in total plant fresh weights were amounted to $18.70 \% \& 19.81 \%$ and $24.44 \%$ and associated with $R$ factor values of $1.6,1.8$, and $1.3(R \geq 1)$, respectively.
\end{abstract}

Keywords: Meloidogyne incognita, malvaceae cultivars, cotton, okra and rosella.

\section{INTRODUCTION}

Plants related to the family malvaceae are widely varied, and includes such important crops i.e. cotton, okra, and rosella, which mainly used as sources of fiber, food and beverages, medicines, timber, and in horticulture (gardening). Cotton, Gossypium barbadense is the world leading fiber crop and it is the second most important oilseed crops. It is known as the "White Gold" and economically important crop. Okra, Abelmoschus esculentus L. is one of the most important vegetables grown throughout tropics and sub-tropics. The rosella, Hibiscus sabdariffa L. is a species of Hibiscus native to the Old World tropics, used for the production of best fiber and as an infusion. Roselle is a medicinal plant in the malvaceae family that originated in Egypt. Hibiscus is considered to be a tropical plant that grows annually. All the parts of Hibiscus Sabdariffa L. are used for medicinal purposes, especially in alternative medicine.

Plant parasitic nematodes, i.e Meloidogyne spp. caused significant damage and losses to most agricultural crops in the tropical and sub-tropical (Luc et. al., 2005). Of the root-knot nematodes, $M$. incognita (Kofoide\& White) chitwood is considered to be the most popular species which affect major field and vegetable crops and caused great economic damages.

The suitability of a host for plant-parasitic nematode is expressed as the ability of the nematode to multiply on the plant. Host suitability may be expressed objectively as the ratio of the number of nematode units recovered at the end of the test, the final nematode population density (Pf), to the number of nematode units used to inoculate the plant, the initial population density (Pi) (Lewis, 1987). It is well known that plants reacted differently to various nematodes. Moreover, this work was carried out to test certain cultivars of malvaceae plants i.e. cotton, okra and rosella suitability to root knot nematodes Meloidogyne incognita infection under greenhouse conditions $\left(29 \pm 4{ }^{\circ} \mathrm{C}\right)$.

\section{MATERIALS AND METHODS}

Source of Nematodes: Second stage juveniles (J2) of Meloidogyne incognita (Kofoid \& White) Chitwood were obtained from a pure culture of $M$. incognita that was initiated by single eggmass and 
propagated on coleus plants, Coleus blumei in the greenhouse of Nematology Research Unit, Agricultural Zoology Department, Faculty of Agriculture, Mansoura University, Egypt, where this work was carried-out.

Host suitability of certain Malvaceae cultivars to the root knot nematode Meloidogyne incognita under greenhouse conditions at $29 \pm 4$ -C: A greenhouse experiment was conducted in order to evaluate nine Malvaceae cultivars namely: cotton, Gossypium barbadense (four cultivars i.e. Giza 70, Giza 86, Giza 89 and Giza 90), okra, Abelmoschus esculentus (three cultivars i.e. Hyper Dokki 1, Hyper Dokki 2 and Ismaily) and rosella, Hibiscus sabdariffa (two cultivars i.e. Hardy and Sabahia 17) for their susceptibility to the root knot nematode, $M$. incognita infection. Seventy two plastic pots $10-\mathrm{cm}-\mathrm{d}$ filled with $900 \mathrm{~g}$ steam sterilized sandy loam soil (1:1) (v:v) were used in this experiment. Seeds of each cultivar were sown and thinned at the rate of one plant per each plastic pot. After 15 days, eight pots were used for each plant cultivar, where four of them were inoculated with 1700 (J2) of M. incognita after 15 days from germination, while the other four pots were left free of nematode inoculum to serve as control. The inocula were introduced to plants by pippeting the inoculum suspension in three holes made around the seedlings covered with sand and irrigated gently with tap water.

All pots were arranged at a randomized complete block design on a bench of a greenhouse at $29 \pm 4$ ${ }^{\circ} \mathrm{C}$. Plants were watered and regularly receiving conventional pesticides against mites and insects as needed and horticulturally treated the same.

Plants were harvested after 45 days from nematode inoculation, and plant growth criteria i.e. shoot and root lengths and fresh weights, as well as shoot dry weight were determined and recorded. Number of $M$. incognita $\mathrm{J}_{2}$ in $250 \mathrm{~g}$. soil/pot were extracted by sieving and modified Baermann- technique (Goodey, 1957), then calculated for each pot, counted by Hawksely counting slide under $\times 10$ magnification and recorded. Infected roots of each plant were washed with tap water, fixed in $4 \%$ formalin for 24 hrs and stained in 0.01 lactic acid-fuchsin (Byrd et al., 1983) and then examined for the number of galls, developmental stages, females and eggmasses.
Host category based on the relationship between host growth response (\% reduction in total plant fresh weight) and reproduction factor (RF) as follows: $0-10 \%$ reduction in plant growth; $R F=0$ Immune (I), RF $<1$ Resistant (R), RF $>1$ Tolerant (T); $11-30 \%$ reduction in plant growth; $R F<1$ Moderately Resistant (MR), RF $>1$ Susceptible (S); and $30 \%<$ reduction in plant growth; $R F<1$ Intolerant (IT), RF>1 Highly Susceptible (HS).

Data were subjected to analysis of variance (ANOVA) (Gomez and Gomez, 1984) followed by Duncan's multiple range test to compare means (Duncan, 1955).

\section{RESULTS AND DISCUSSION}

Data in Table (1) and Fig.(1) represent the host suitability of nine Malvaceae cultivars i.e. cotton, $G$. barbadense (4 cultivars i.e. Giza 89, Giza 70, Giza 90 and Giza 86), okra, $A$. esculentus (three cultivars i.e. Hyper Dokki 1, Hyper Dokki 2 and Ismaily) and rosella, H. sabdariffa (two cultivars i.e. Hardy and Sabahia 17) as affected by $M$. incognita infection under greenhouse conditions. Results revealed that plant growth of all tested Malvaceae cultivars were affected by nematode infection at various degrees. Among tested cotton cultivars, cv. Giza 90 accomplished the highest reduction values in total fresh weight $(36.19 \%)$, plant length $(32.71 \%)$ and shoot dry weight (39.72\%), followed by cv, Giza 86 with values of $34.32,31.96$ and $37.63 \%$ for the same plant growth parameters, respectively, whereas their least values were recorded by cotton cvs. Giza 89\& Giza 70 which were amounted to be $18.70,24.71$ and $34.05 \%$; and $19.81,24.80$ and $35.38 \%$, respectively. With respect to okra cultivars, Hyper Dokki 1 gave the highest reduction percentage for the same plant parameters with values of $32.41,37.74$ and 43.57, whereas their least values recorded by okra cv. Ismaily that were amounted to $21.81,8.56$ and $21.76 \%$ for total plant fresh weight, total plant length and shoot dry weight, respectively. Meanwhile, among two tested rosella cultivars, Sabahia 17 showed the highest reduction percentage for the same plant growth criteria with values of $31.54,20.66$ and $38.74 \%$ whereas, their least values were recorded by rosella cv. Hardy with values of 31.30 , 5.58 and $27.81 \%$ for total plant fresh weight, total plant length and shoot dry weight, respectively.(Table 1 \& Fig. 1). 
Table (1): Plant growth response of three Malvaceae plant cultivars to $M$. incognita infection under greenhouse conditions $(29 \pm 4 \stackrel{\circ}{\circ})$.

\begin{tabular}{|c|c|c|c|c|c|c|c|c|c|c|c|}
\hline \multicolumn{2}{|c|}{ Treatments } & \multicolumn{10}{|c|}{ Plant growth response } \\
\hline \multirow{2}{*}{ 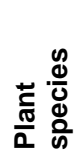 } & \multirow[t]{2}{*}{ Cultivar } & \multicolumn{2}{|c|}{ Length (cm) } & \multirow{2}{*}{$\begin{array}{l}\text { Total } \\
\text { length }\end{array}$} & \multirow[t]{2}{*}{$\begin{array}{c}\text { * } \\
\text { Red. \% }\end{array}$} & \multicolumn{2}{|c|}{$\begin{array}{c}\text { Fresh weight } \\
(\mathrm{g})\end{array}$} & \multirow{2}{*}{$\begin{array}{c}\text { Fresh } \\
\text { weight } \\
\text { of } \\
\text { whole } \\
\text { plant }\end{array}$} & \multirow{2}{*}{$\begin{array}{c}* \\
\text { Red. } \\
\%\end{array}$} & \multirow{2}{*}{$\begin{array}{c}\text { Shoot } \\
\text { dry } \\
\text { weight } \\
\text { (g) }\end{array}$} & \multirow[t]{2}{*}{$\begin{array}{c}* \\
\text { Red. \% }\end{array}$} \\
\hline & & Shoot & Root & & & Shoot & Root & & & & \\
\hline \multirow{8}{*}{ 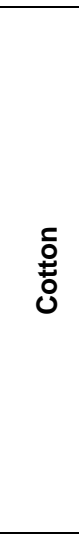 } & Giza 70 & $\begin{array}{c}32.0 \\
d\end{array}$ & $\begin{array}{c}30.5 \\
a\end{array}$ & $\begin{array}{l}62.5 \\
\text { bcd }\end{array}$ & - & $\begin{array}{l}4.48 \\
\text { bc }\end{array}$ & $\begin{array}{c}3.80 \\
\text { bc }\end{array}$ & $\begin{array}{c}8.28 \\
b\end{array}$ & - & $\begin{array}{c}3.42 \\
\mathrm{a}\end{array}$ & - \\
\hline & Giza $70+N$ & $\begin{array}{c}26.5 \\
e\end{array}$ & $\begin{array}{c}20.5 \\
\text { bd }\end{array}$ & $\begin{array}{l}47.0 \\
\text { def }\end{array}$ & 24.80 & $\begin{array}{c}3.67 \\
\text { cd }\end{array}$ & $\begin{array}{c}2.97 \\
\text { de }\end{array}$ & $\begin{array}{l}6.65 \\
\text { cde }\end{array}$ & 19.81 & $\begin{array}{c}2.21 \\
c d\end{array}$ & 35.38 \\
\hline & Giza 86 & $\begin{array}{l}34.7 \\
\mathrm{~cd}\end{array}$ & $\begin{array}{l}28.5 \\
a b\end{array}$ & $\begin{array}{c}63.20 \\
\text { bc }\end{array}$ & - & $\begin{array}{c}4.96 \\
a\end{array}$ & $\begin{array}{c}3.46 \\
\mathrm{C}\end{array}$ & $\begin{array}{c}8.43 \\
\text { bc }\end{array}$ & - & $\begin{array}{c}2.79 \\
\text { bc }\end{array}$ & - \\
\hline & Giza $86+N$ & $\begin{array}{l}24.5 \\
\mathrm{fg}\end{array}$ & $\begin{array}{c}18.50 \\
d\end{array}$ & $\begin{array}{c}43.0 \\
f\end{array}$ & 31.96 & $\begin{array}{c}2.82 \\
\text { C }\end{array}$ & $\begin{array}{c}2.71 \\
f\end{array}$ & $\begin{array}{c}5.53 \\
d\end{array}$ & 34.32 & $\begin{array}{c}1.74 \\
\text { de }\end{array}$ & 37.63 \\
\hline & Giza 89 & $\begin{array}{c}38.75 \\
\text { bc }\end{array}$ & $\begin{array}{c}27.0 \\
\text { b }\end{array}$ & $\begin{array}{c}65.75 \\
a b\end{array}$ & - & $\begin{array}{l}4.32 \\
\text { bcd }\end{array}$ & $\begin{array}{c}3.86 \\
b\end{array}$ & $\begin{array}{c}8.19 \\
C\end{array}$ & - & $\begin{array}{c}2.32 \\
\text { C }\end{array}$ & - \\
\hline & Giza $89+N$ & $\begin{array}{c}25.0 \\
\text { ef }\end{array}$ & $\begin{array}{c}24.5 \\
\text { c }\end{array}$ & $\begin{array}{c}49.5 \\
\text { de }\end{array}$ & 24.71 & $\begin{array}{c}3.54 \\
e\end{array}$ & $\begin{array}{c}3.11 \\
d\end{array}$ & $\begin{array}{c}6.66 \\
\mathrm{~cd}\end{array}$ & 18.70 & $\begin{array}{c}1.53 \\
\mathrm{e}\end{array}$ & 34.05 \\
\hline & Giza 90 & $\begin{array}{c}41.0 \\
a\end{array}$ & $\begin{array}{l}25.5 \\
\text { bc }\end{array}$ & $\begin{array}{c}66.5 \\
a\end{array}$ & - & $\begin{array}{l}4.85 \\
a b\end{array}$ & $\begin{array}{c}4.13 \\
a\end{array}$ & $\begin{array}{c}8.98 \\
a\end{array}$ & - & $\begin{array}{c}2.82 \\
b\end{array}$ & - \\
\hline & Giza $90+N$ & $\begin{array}{c}26.0 \\
e\end{array}$ & $\begin{array}{c}18.75 \\
d\end{array}$ & $\begin{array}{c}44.75 \\
\text { ef }\end{array}$ & 32.71 & $\begin{array}{c}3.43 \\
\text { ef }\end{array}$ & $\begin{array}{c}2.3 \\
\mathrm{~g}\end{array}$ & $\begin{array}{c}5.73 \\
f\end{array}$ & 36.19 & $\begin{array}{c}1.70 \\
\mathrm{~d}\end{array}$ & 39.72 \\
\hline \multirow{6}{*}{ 章 } & Ismaily & $\begin{array}{c}44.0 \\
b\end{array}$ & $\begin{array}{c}29.0 \\
b\end{array}$ & $\begin{array}{c}73.0 \\
b\end{array}$ & - & $\begin{array}{c}7.66 \\
\text { bc }\end{array}$ & $\begin{array}{c}4.08 \\
b c\end{array}$ & $\begin{array}{c}11.74 \\
c\end{array}$ & - & $\begin{array}{c}4.09 \\
a b\end{array}$ & - \\
\hline & Ismaily +N & $\begin{array}{c}38.75 \\
d\end{array}$ & $\begin{array}{c}28.0 \\
\text { c }\end{array}$ & $\begin{array}{c}66.75 \\
\mathrm{~d}\end{array}$ & 8.56 & $\begin{array}{c}6.23 \\
d\end{array}$ & $\begin{array}{c}2.95 \\
\mathrm{de}\end{array}$ & $\begin{array}{c}9.19 \\
d\end{array}$ & 21.81 & $\begin{array}{l}3.2 \\
\text { bc }\end{array}$ & 21.76 \\
\hline & Hyper Dokki 1 & $\begin{array}{c}43.0 \\
c\end{array}$ & $\begin{array}{c}21.25 \\
\mathrm{e}\end{array}$ & $\begin{array}{c}64.25 \\
\mathrm{e}\end{array}$ & - & $\begin{array}{c}8.41 \\
b\end{array}$ & $\begin{array}{c}4.61 \\
\mathrm{a}\end{array}$ & $\begin{array}{c}13.02 \\
b\end{array}$ & - & $\begin{array}{c}4.59 \\
\mathrm{a} \\
\end{array}$ & - \\
\hline & $\begin{array}{l}\text { Hyper Dokki } \\
1+N\end{array}$ & $\begin{array}{c}20.0 \\
\mathrm{e}\end{array}$ & $\begin{array}{c}20.0 \\
f\end{array}$ & $\begin{array}{c}40.0 \\
f\end{array}$ & 37.74 & $\begin{array}{c}5.94 \\
e\end{array}$ & $\begin{array}{c}2.86 \\
\mathrm{e}\end{array}$ & $\begin{array}{c}8.8 \\
e\end{array}$ & 32.41 & $\begin{array}{c}2.59 \\
\mathrm{e}\end{array}$ & 43.57 \\
\hline & Hyper Dokki 2 & $\begin{array}{c}50.75 \\
a \\
\end{array}$ & $\begin{array}{c}36.25 \\
\mathrm{a}\end{array}$ & $\begin{array}{c}87.0 \\
a\end{array}$ & - & $\begin{array}{c}10.36 \\
a \\
\end{array}$ & $\begin{array}{c}4.33 \\
b\end{array}$ & $\begin{array}{c}14.69 \\
\mathrm{a}\end{array}$ & - & $\begin{array}{c}3.63 \\
\text { b }\end{array}$ & - \\
\hline & $\begin{array}{l}\text { Hyper Dokki } \\
2+\mathrm{N}\end{array}$ & $\begin{array}{c}43.75 \\
\text { bc }\end{array}$ & $\begin{array}{c}25.0 \\
d\end{array}$ & $\begin{array}{c}68.75 \\
\mathrm{C} \\
\end{array}$ & 20.98 & $\begin{array}{c}7.27 \\
\text { C }\end{array}$ & $\begin{array}{c}3.83 \\
d\end{array}$ & $\begin{array}{c}11.10 \\
\text { cd }\end{array}$ & 24.44 & $\begin{array}{c}2.78 \\
d\end{array}$ & 23.42 \\
\hline \multirow{4}{*}{ 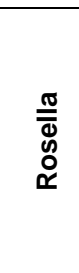 } & Hardy & $\begin{array}{c}25.25 \\
a\end{array}$ & $\begin{array}{c}24.0 \\
b\end{array}$ & $\begin{array}{c}49.25 \\
b\end{array}$ & - & $\begin{array}{c}8.50 \\
a\end{array}$ & $\begin{array}{c}4.60 \\
b\end{array}$ & $\begin{array}{c}13.11 \\
a\end{array}$ & - & $\begin{array}{c}3.2 \\
\mathrm{a}\end{array}$ & - \\
\hline & Hardy + N & $\begin{array}{c}24.0 \\
b\end{array}$ & $\begin{array}{c}22.5 \\
\text { c }\end{array}$ & $\begin{array}{c}46.5 \\
\mathrm{c}\end{array}$ & 5.58 & $\begin{array}{c}6.04 \\
b\end{array}$ & $\begin{array}{c}2.96 \\
\mathrm{~d}\end{array}$ & $\begin{array}{c}9.01 \\
b c\end{array}$ & 31.30 & $\begin{array}{c}2.31 \\
b\end{array}$ & 27.81 \\
\hline & Sabahia 17 & $\begin{array}{c}23.5 \\
\mathrm{C}\end{array}$ & $\begin{array}{c}30.7 \\
a\end{array}$ & $\begin{array}{c}54.20 \\
a\end{array}$ & - & $\begin{array}{c}4.81 \\
\mathrm{C}\end{array}$ & $\begin{array}{c}5.62 \\
a\end{array}$ & $\begin{array}{c}10.43 \\
b\end{array}$ & - & $\begin{array}{c}2.22 \\
b c\end{array}$ & - \\
\hline & Sabahia $17+N$ & $\begin{array}{c}22.25 \\
d\end{array}$ & $\begin{array}{c}20.75 \\
d\end{array}$ & $\begin{array}{c}43.0 \\
d\end{array}$ & 20.66 & $\begin{array}{c}3.83 \\
d\end{array}$ & $\begin{array}{c}3.31 \\
\mathrm{C}\end{array}$ & $\begin{array}{c}7.14 \\
\text { c } \\
\end{array}$ & 31.54 & $\begin{array}{c}1.36 \\
\mathrm{c}\end{array}$ & 38.74 \\
\hline
\end{tabular}

$\mathrm{N}=1700 \mathrm{~J} 2$ of $M$. incognita

Each value is a mean of four replicates.

Means in each column followed by the same letter(s) did not differ at $p \leq 0.05$ according to Duncan's multiplerange test.

${ }^{*}$ Reduction $\%=\frac{\text { Control (without N) }-\mathrm{N}}{\text { Contol }} \times 100$

$$
\text { Control }
$$


Agric. Biol. J. N. Am., 2013, 4(3): 309-315

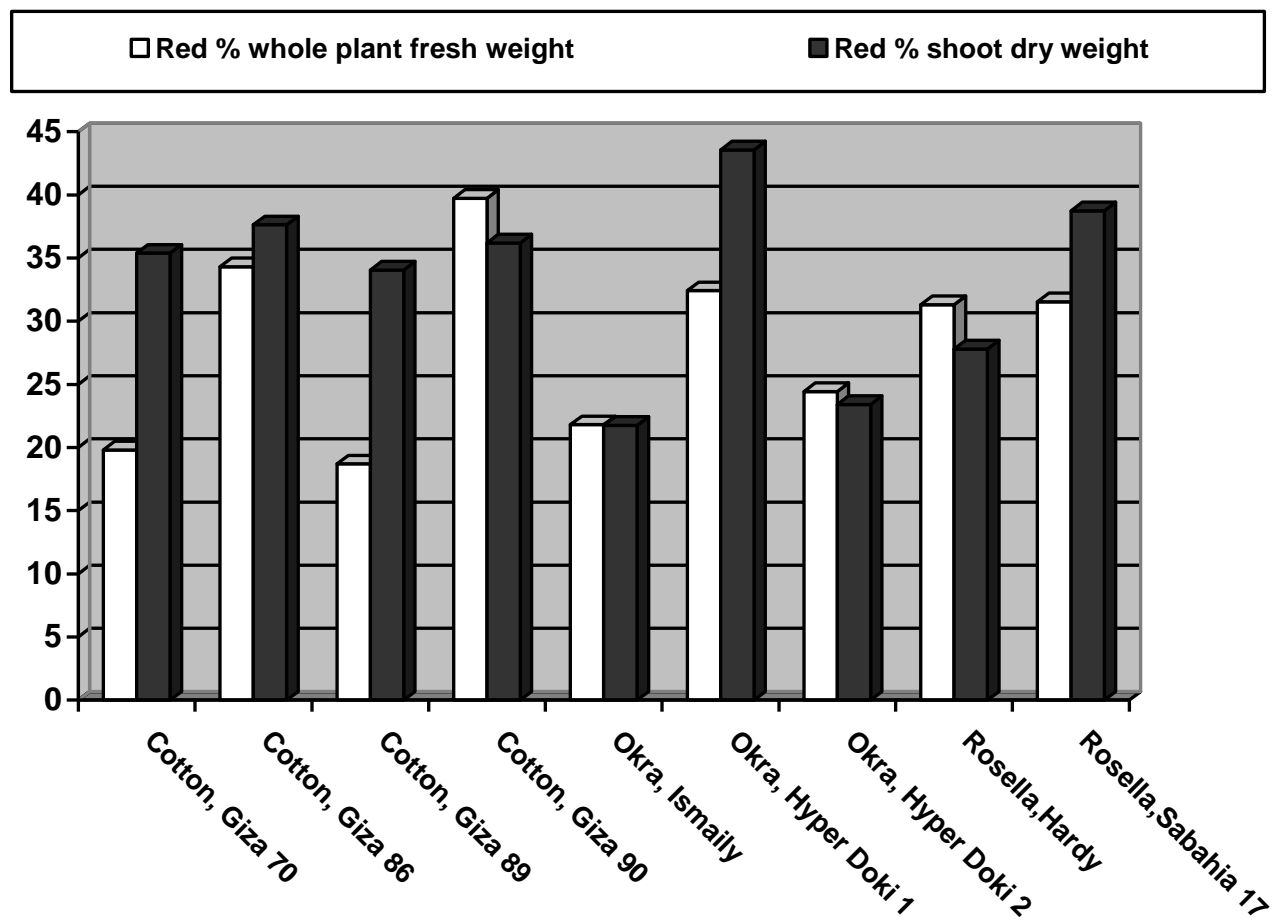

Fig.(1): Reduction percentage of whole plant and shoot dry weights of three Malvaceae plant cultivars to $M$. incognita infection under greenhouse conditions $\left(29 \pm 4^{\circ} \mathrm{C}\right)$.

The screening of Malvaceae plant cultivars revealed that none of the tested cultivars were immune to rootknot nematode, $M$. incognita, though the incidence varied from cultivar to another. Evaluated Malvaceae plant cultivars differed greatly in their abilities to support $M$. incognita populations (Table 2 \& Fig 2). Of the screened nine plant cultivars, okra cv. Hyper Dokki 1 exhibited the highest rate of nematode reproduction $(\mathrm{RF}=3.1)$ followed by cotton cv. Giza 86 (2.5), Giza 90 (2.0) and rosella cv. sabahia 17 and okra cv. Hyper Dokki 2 (1.3) each, respectively.

Moreover, number of galls and egg-masses recovered from roots differed among tested plant species. Likewise, the greatest number of galls were recorded on okra cv. Hyper Dokki 1, cotton cv. Giza 86 and rosella cv. Sabahia 17 , with root gall indices (RGI) 4.6, 4.5 and 2.2, respectively. Similar trend was noticed with number of egg-masses for the same cultivar species with egg-masses index (EI) 4.4, 4.4 and 2.1, respectively.

Host category at the tested Malvaceae plants was determined based on the relationship between host plant growth response (\% reduction of total plant fresh weight) and nematode reproduction factor (RF), (Table 3 ):-

- $\quad$ Cotton cvs. Giza86, Giza 90 , okra cv. Hyper Dokki 1 and rosella cv Sabahia 17 were rated as highly susceptible hosts (HS) since plant growth was highly affected with reproduction factor $>1$.

- Cotton cvs. Giza 89, Giza 70 and okra cv. Hyper Dokki 2 were rated as susceptible hosts (S) since plant growth was moderately affected with reproduction factor $>1$.

- Rosella cv. Hardy was rated as intolerant host (IT) since plant growth was highly affected with reproduction factor $<1$.

- Finally, okra cv. Ismaily was rated as moderately resistant host (MR) since plant growth moderately affected and reproduction factor $(\mathrm{RF})<1$.

Moreover, regression analysis between nematode reproduction factor and nine Malvaceae cultivars gave $R^{2}$ value of 0.1535 (Fig. 2). 
Agric. Biol. J. N. Am., 2013, 4(3): 309-315

Table (3): Host suitability of nine Malvaceae cultivars within three plant species to $M$. incognita infection under greenhouse conditions $(29 \pm 4 \stackrel{\circ}{ })$.

\begin{tabular}{|c|c|c|c|c|c|c|c|c|c|c|}
\hline \multicolumn{2}{|c|}{ Treatments } & \multicolumn{4}{|c|}{${ }^{\star}$ Nematode population in } & \multirow{3}{*}{ 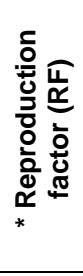 } & \multirow{3}{*}{ 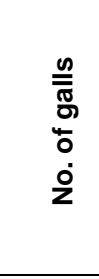 } & \multirow{3}{*}{$\begin{array}{c}\star \star \\
\text { RGI }\end{array}$} & \multirow{3}{*}{ 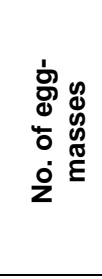 } & \multirow{3}{*}{$\begin{array}{l}* * * \\
\text { EI }\end{array}$} \\
\hline \multirow{2}{*}{$\begin{array}{l}=\frac{0}{0} \\
\frac{\pi}{\alpha} \frac{0}{\infty}\end{array}$} & \multirow[b]{2}{*}{ Cultivar } & \multirow[b]{2}{*}{ Soil/pot } & \multicolumn{2}{|c|}{ Root } & \multirow{2}{*}{$\begin{array}{c}\text { Total } \\
\text { (Pf) }\end{array}$} & & & & & \\
\hline & & & $\begin{array}{c}\text { Develop. } \\
\text { stages }\end{array}$ & Females & & & & & & \\
\hline \multirow{4}{*}{$\begin{array}{l}\text { o } \\
\text { ثે } \\
\text { o }\end{array}$} & Giza 70 & $3020.0 \mathrm{c}$ & $17.0 \mathrm{~b}$ & $45.0 \mathrm{~b}$ & $3082.0 \mathrm{c}$ & 1.8 & $55.0 \mathrm{~b}$ & 4.4 & $33 \mathrm{~b}$ & 4.2 \\
\hline & Giza 86 & $4125.0 \mathrm{a}$ & $34.0 \mathrm{ab}$ & $50.0 \mathrm{a}$ & $4209.0 \mathrm{a}$ & 2.5 & $59.0 \mathrm{a}$ & 4.5 & $46.0 \mathrm{a}$ & 4.4 \\
\hline & Giza 89 & $2697.0 \mathrm{~d}$ & $16.0 \mathrm{c}$ & $26.0 \mathrm{~d}$ & $2739.0 \mathrm{~d}$ & 1.6 & $28.0 \mathrm{~d}$ & 3.2 & $22.0 \mathrm{~d}$ & 3.1 \\
\hline & Giza 90 & $3256.0 \mathrm{~b}$ & $36.0 \mathrm{a}$ & $30.0 \mathrm{c}$ & $3322.0 b$ & 2.0 & $36.0 \mathrm{c}$ & 4.3 & $25.0 \mathrm{c}$ & 3.2 \\
\hline \multirow{3}{*}{ 芇 } & Ismaily & $1478.0 \mathrm{c}$ & $22.0 \mathrm{~b}$ & $20.0 \mathrm{~b}$ & $1520.0 \mathrm{c}$ & 0.9 & $10.0 \mathrm{c}$ & 2.2 & $7.0 \mathrm{c}$ & 2.0 \\
\hline & Hyper Dokki 1 & $5240.0 \mathrm{a}$ & $51.5 \mathrm{a}$ & $52.0 \mathrm{a}$ & $5343.5 a$ & 3.1 & $76.0 \mathrm{a}$ & 4.6 & $49.5 \mathrm{a}$ & 4.4 \\
\hline & Hyper Dokki 2 & $2225.0 \mathrm{~b}$ & $13.5 \mathrm{c}$ & $20.0 \mathrm{~b}$ & $2258.5 b$ & 1.3 & $29.0 \mathrm{~b}$ & 3.3 & $15.5 \mathrm{~b}$ & 3.2 \\
\hline \multirow{2}{*}{ 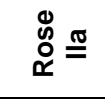 } & Hardy & $1515.7 \mathrm{~b}$ & $1.75 \mathrm{~b}$ & $7.25 \mathrm{~b}$ & $1524.7 \mathrm{~b}$ & 0.9 & $9.5 \mathrm{~b}$ & 2.1 & $2.25 \mathrm{~b}$ & 1.1 \\
\hline & Sabahia 17 & $2165.2 \mathrm{a}$ & $9.5 \mathrm{a}$ & $10.75 \mathrm{a}$ & $2185.5 a$ & 1.3 & $10.0 \mathrm{a}$ & 2.2 & $5.75 \mathrm{a}$ & 2.1 \\
\hline
\end{tabular}

$\mathrm{N}=1700 \mathrm{~J} 2$ of $M$. incognita $(\mathrm{Pi})$

Each value is a mean of four replicates.

** Root gall index (RGI) or eggmass index (EI): $0=$ no galling or egg-masses , $1=1-2$ galls or egg-masses ; $2=3-10$ galls or egg-masses ; $3=11$-30 galls or egg-masses ; $4=31-100$ galls or egg-masses and $5=$ more than 100 galls or egg-masses ... Talyor and Sasser,1978).

Means in each column followed by the same letter(s) significantly are not different $(\mathrm{P} \leq 0.05)$ by Duncan's multiple range test.

${ }^{*}$ Reproduction Factor $=$ Final population $(\mathrm{Pf})$ Initial population $(\mathrm{Pi})$

Reproductin factor

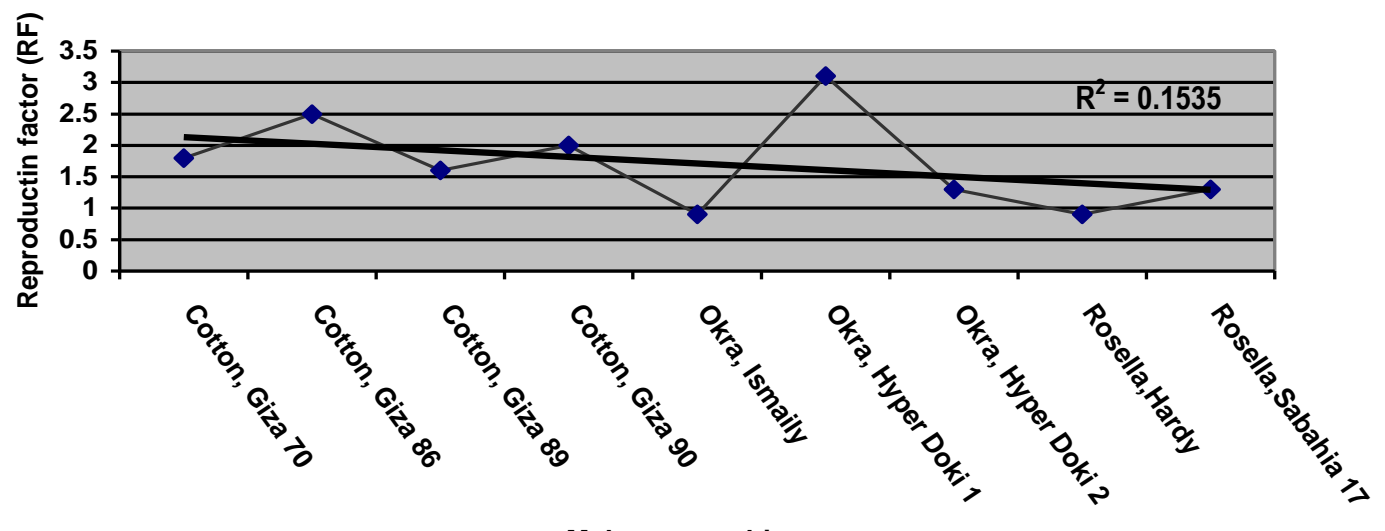

Malvaceae cultivars

Fig. (2): Reproduction factor of $M$. incognita infecting nine Malvaceae cultivars within three plant species under greenhouse conditions $(29 \pm 4 \stackrel{\circ}{\circ})$. 
Table (3): Relative susceptibility of nine Malvaceae cultivars within three plant species to $M$. incognita infection.

\begin{tabular}{|c|c|c|c|c|c|c|}
\hline 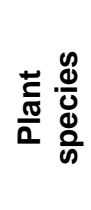 & Cultivar & $\begin{array}{c}\text { Fresh } \\
\text { weight } \\
\text { Red. } \\
\%\end{array}$ & 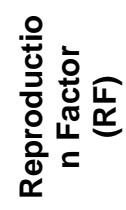 & RGI & 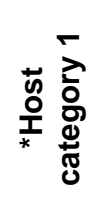 & 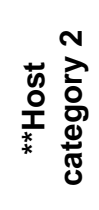 \\
\hline \multirow{4}{*}{$\begin{array}{l}\text { o } \\
\text { ثे }\end{array}$} & Giza 70 & 19.81 & 1.8 & 4.4 & $S$ & $\mathrm{~s}$ \\
\hline & Giza 86 & 34.32 & 2.5 & 4.5 & HS & $\mathrm{s}$ \\
\hline & Giza 89 & 18.7 & 1.6 & 3.2 & $\mathbf{S}$ & $\mathbf{S}$ \\
\hline & Giza 90 & 36.19 & 2.0 & 4.3 & HS & $\mathrm{S}$ \\
\hline \multirow{3}{*}{$\frac{\pi}{\frac{\pi}{2}}$} & Ismaily & 21.81 & 0.9 & 2.2 & MR & S \\
\hline & Hyper Dokki 1 & 32.41 & 3.1 & 4.6 & HS & $\mathrm{s}$ \\
\hline & Hyper Dokki 2 & 24.44 & 1.3 & 3.3 & s & $\mathrm{s}$ \\
\hline \multirow{2}{*}{ 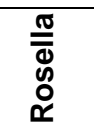 } & Hardy & 31.3 & 0.9 & 2.1 & IT & $\mathbf{R}$ \\
\hline & Sabahia 17 & 31.54 & 1.3 & 2.2 & HS & $\mathbf{S}$ \\
\hline
\end{tabular}

*Host category (1) based on the relationship between host response and $\mathrm{R}$ factor as follows:

$0-10 \%$ reduction in plant growth; $\mathrm{RF}=0$ Immune $(\mathrm{I})$

$$
\mathrm{RF}<1 \text { Resistant }(\mathrm{R})
$$

$\mathrm{RF}>1$ Tolerant $(\mathrm{T})$.

$11-30 \%$ reduction in plant growth; $R F<1$ moderately Resistant (MR)

$\mathrm{RF}>1$ Susceptible (S).

$30 \%<$ reduction in plant growth; $\mathrm{RF}<1$ Intolerant (IT)

$\mathrm{RF}>1$ Highly Susceptible (HS).

**Host category (2)based on root gall index (RGl) according to Canto-Saez and Brodie (1987) as follows:

$(R G I \leq 2 \& R F \leq 1)$ resistant $(R),(R G I \leq 2 \& R F>1)$ tolerant $(T)$ and (RGI $\geq 2 \& R F \geq 1)$ susceptible (S).

Apparently, concerning the present results on host suitabilities of certain Malvaceae plant cultivars i.e. cotton cvs. Giza 89, Giza 70, Giza 90 and Giza 86; okra, cvs. Hyper Dokki 1, Hyper Dokki 2 and Ismaily; and rosella, cvs. Hardy and Sabahia 17 to $M$. incognita infection under greenhouse conditions, that based on the relationship between host plant response in terms of reduction percentage in plant growth as indicator of plant damage and reproduction factor (RF) as indicator of nematode build-up or host efficiency indicated that non of the tested Malvaceae plant cultivars were immune to $M$. incognita infection, while okra cv. Ismaily and rosella cv. Hardy were classified as moderately resistant and intolerant host, respectively, whereas cotton cvs. Giza 90 and Giza 86 as well as okra cv. Hyper Dokki 1 and rosella cv. Sabahia 17 were rated as highly susceptible hosts.
Moreover, cotton cv. Giza 89 and Giza 70; and okra cv. Hyper Dokki 2 were considered as susceptible hosts as well, respectively. However, when the relationship between root gall index as indicator of plant damage and rate of nematode increase ( $R$ factor) as indicator of nematode reproduction or host efficiency were used (Canto-Saenz and Brodie 1987), different host categories in this work was detected, since all tested cotton cvs. Giza 89, Giza 70 , Giza 90 and Giza 86; and okra cvs. Ismaily, Hyper Dokki 1\& Hyper Dokki 2 were rated as susceptible hosts, whereas rosella cv. Hardy was classified as resistant host, while rosella cv. Sabahia 17 was ranked as susceptible host to $M$. incognita infection. Moreover, results of the present findings disagreed with those reported by El-Sherif et al.(2007a) who stated that cotton cv. 89 was scored 
as resistant and agreed with their results in respect to cotton cv. Giza 86 as susceptible host to $M$. incognita infection, since their root gall indices were $2 \& 4$ with $R$ factor values of 0.4 and 1.11 , respectively. Meanwhile, okra cv. Ismaily was classified as moderately resistant host to $M$. incognita, so herein it could be recommended to the growers for commercial okra cultivation, while other cultivars such as cotton cvs. Giza 89, Giza 70, Giza 90, Giza 89, okra cvs. Hyper Dokki 1 \& Hyper Dokki 2 , and rosella cvs. Hardy and Sabahia 17 that were damaged and considered as susceptible hosts for $M$. incognita infection, should be eliminated from field production. Furthermore, the usefulness of these cultivars in crop rotation schemes in contaminated soil with Meloidogyne spp. is very limited because none of the tested cultivars showed high degree of resistance to such nematode infection

\section{REFERENCES}

Byrd, D.W.; T. Kirkpatrick and K. Barker (1983). An improved technique for clearing and staining plant tissues for detection nematodes. J. Nematol., 15 (3): 142-143.
Canto- Saenz, M. and B. B. Brodie (1987). Comparison of compatible and incompatible response of potato to Meloidogyne incognita. J. Nematol. 19(2):218-221.

Duncan, D.B. (1955). Multiple range and multiple, F-test. Biometrics, 11: 1-42.

El-sherif, A. G. , A. R. Refaei and A. E. M. Khalil(2007). Status of plant parasitic nematodes associated with cotton filed in North Eastern Nile Delta Region, Egypt with special reference to host suitability to Meloidogyne incognita infection. J. Agric . Mansoura Univ., 32(10).: 8695-8709.

Gomez, K. A. and A.A. Gomez (1984). Statistical procedures for Agricultural Research. 2nd Ed., John Wiley \&Sons: Inc., New York.

Goodey, J.B. (1957). Laboratory methods for work with plant and soil nematodes. Tech. Bull. No. 2. Min. Agric. Fish Ed. London, pp 47.

Lewis, S. A. (1987). Nematode-plant compatibility in Veech, A. \& Dickson, D. W. (Eds). Vistas on Nematology. Hyattsville, MD. USA, Society of Nematologists, pp. 246-252.

Luc, M., R. A. Sikora, and J.Bridge (2005). Plant Parasitic Nematodes in subtropical and tropical Agriculture. $2^{\text {nd }}$ edn. CAB international, Wallingford, Uk.

Taylor, A. L. and Sasser, J. N. (1978).Biology identification and control of root-knot nematode(Meloidogyne spp.) Raleigh:North Carolina state University Graphics. 